\title{
Business Process Re-engineering (BPR): The REBUS Approach
}

V.Hlupic, J. Choudrie, and N.Patel

Department of Information Systems and Computing, Brunel University,

Uxbridge, Middlesex. UB8 3PH. United Kingdom.

Tel: $+44(0) 1895203259$

Fax: $+44(0) 1895251686$

\{Vlatka.Hlupic, Jyoti.Choudrie and Nayna.Patel@brunel.ac.uk\}

\begin{abstract}
Many organisations undertake Business Process Re-engineering (BPR) projects in order to improve efficiency and reduce costs. Although this approach can result in significant improvements and benefits, there are high risks associated with radical changes of business processes and the failure rate of BPR projects is reported to be as high as $70 \%$. The Centre for Re-engineering Business Processes (REBUS) was established at Brunel University to provide a multidisciplinary environment for research into BPR and its success factors. This paper describes the REBUS approach to research concerning the success of business process re-engineering projects and presents examples of some of the projects carried out.
\end{abstract}

Keywords: business process re-engineering, success, human factors; information systems; business process modelling 


\subsection{Introduction}

Organisations are continuously seeking for innovative ways to operate in order to survive in a competitive business environment. Management approaches such as Business Process Re-engineering (BPR) are adopted by many organisations in order to achieve a dramatic increase in performance and cost reduction. As the risks involved and failure rates associated with BPR projects are very high, it is important to investigate the reasons for failures in a systematic and multidisciplinary approach.

The Centre for Re-engineering Business Processes (REBUS) was established in 1997 within the Department of Information Systems and Computing at Brunel University. The researchers working within the centre include academic staff and research students who have a broad range of diverse skills from backgrounds including business, economics, information systems and engineering. It is argued that such diversity supports innovative and interdisciplinary approaches to business process re-engineering (BPR). The research group also includes several academics from institutions in various countries such as Canada, Croatia, Germany and Holland. The primary aim of the Centre is to investigate how the success rate of business process re-engineering can be improved. It is our view that this could be achieved by, for example, reducing resistance to change by considering human and organisational aspects of BPR (Choudrie et al., 1999; Irani and Sharp, 1997; Irani et al., 1997a; Irani et al., 1997b), by reducing the risks of re-engineering by developing models of processes prior to their change (Giaglis et al., 1999c; Hlupic, 1998; Ray et al., ????), and by understanding the role of information technology in BPR (Giaglis and Doukidis, 1998; Giaglis et al., 1999b; Giaglis et al., 1999d). Considering all of these factors in a systematic manner 
can, we suggest, boost the success rate of BPR projects as present literature reports BPR failure rates to be over 50\% (Hammer and Champy, 1995).

In the next section, the management concept of BPR is briefly introduced and the problems associated with this approach are outlined. The REBUS approach to research in BPR is then presented, together with a selection of examples of research projects carried out within the Centre. The paper concludes with the experiences and future anticipated research of members of the research Centre.

\subsection{Business process re-engineering (BPR)}

The increasing competitive pressure that organisations currently face forces them to find ways of minimising the time it takes to develop the product, bring products to the market and offer efficient and effective service to customers whilst at the same time maximising profits. This pressure has made Business Process Re-engineering (BPR) one of the most popular topics in organisational management and has created new ways of doing business (Tumay, 1995). BPR relates to the fundamental rethinking and radical redesign of an entire business system to achieve significant improvements in performance of the company.

Many leading organisations have conducted BPR in order to improve productivity and gain competitive advantage. For example, a survey of 180 US and 100 European companies found that $75 \%$ of these companies had engaged in significant reengineering efforts in the past three years (Jackson, 1996). Amongst the reasons 
leading to the success of BPR is an emphasis on a top-down approach, empowerment, team working and flattening of hierarchies. Nevertheless, despite the success stories associated with BPR, there are high failure rates associated with it. Hammer and Champy (1995) noted that failure rates as high as 70 per cent can be observed as a result of BPR. However presently, data to support this claim is limited.

Research in the area has tried to provide reasons for this high failure rate. Amongst these are: trying to do too much, not appreciating the risk factors (communications, measures, accountability), and not putting the most appropriate people on the project. However, amongst the top five reasons leading to the failure of BPR, the one that concerned people was prominent. In this, 'middle management resistance' was cited as the most common cause for failure. Other reasons contributing to BPR failure were top/senior management, the prevailing culture and political culture, as well as employee fear and resistance to change (Oram and Wellins, 1996). Other frequently cited problems related to business process re-engineering include the inability to predict the outcome of radical change, difficulty in capturing existing processes in a way that can be seen by multidisciplinary team members, a lack of creativity in process redesign, cost of implementing the new process, or inability to recognise the dynamic nature of the processes. It is often argued that one of the major problems that contribute to the failure of BPR projects is a lack of tools for evaluating the effects of designed solutions before implementation (Paolucci et al., 1997; Tumay, 1995).

Mistakes as a result of BPR can only be recognised once the redesigned processes are implemented. This raises important issues, as it is particularly expensive and difficult to attempt the task of correcting earlier mistakes at this point. Although the evaluation 
of alternative solutions might be difficult, it is imperative that the risks associated with BPR projects are minimised. The following section describes the approach that the Centre has adopted to investigate the aforementioned issues.

\subsection{The REBUS approach to BPR}

The Centre for Re-engineering Business Processes at Brunel University aims to investigate how the success rate of business process re-engineering can be improved. We suggest that this can only be achieved by considering all the relevant factors in a systematic manner. Some of these factors include the role of Information Technology in business process change as enabler and implementers, human and organisational factors related to, for example, resistance to change or motivation of teams involved in $\mathrm{BPR}$, and the importance of using dynamic modelling techniques to develop models of processes prior to their change. Some of the current areas of our research are summarised and described in Figure 1 and Table 1 below.

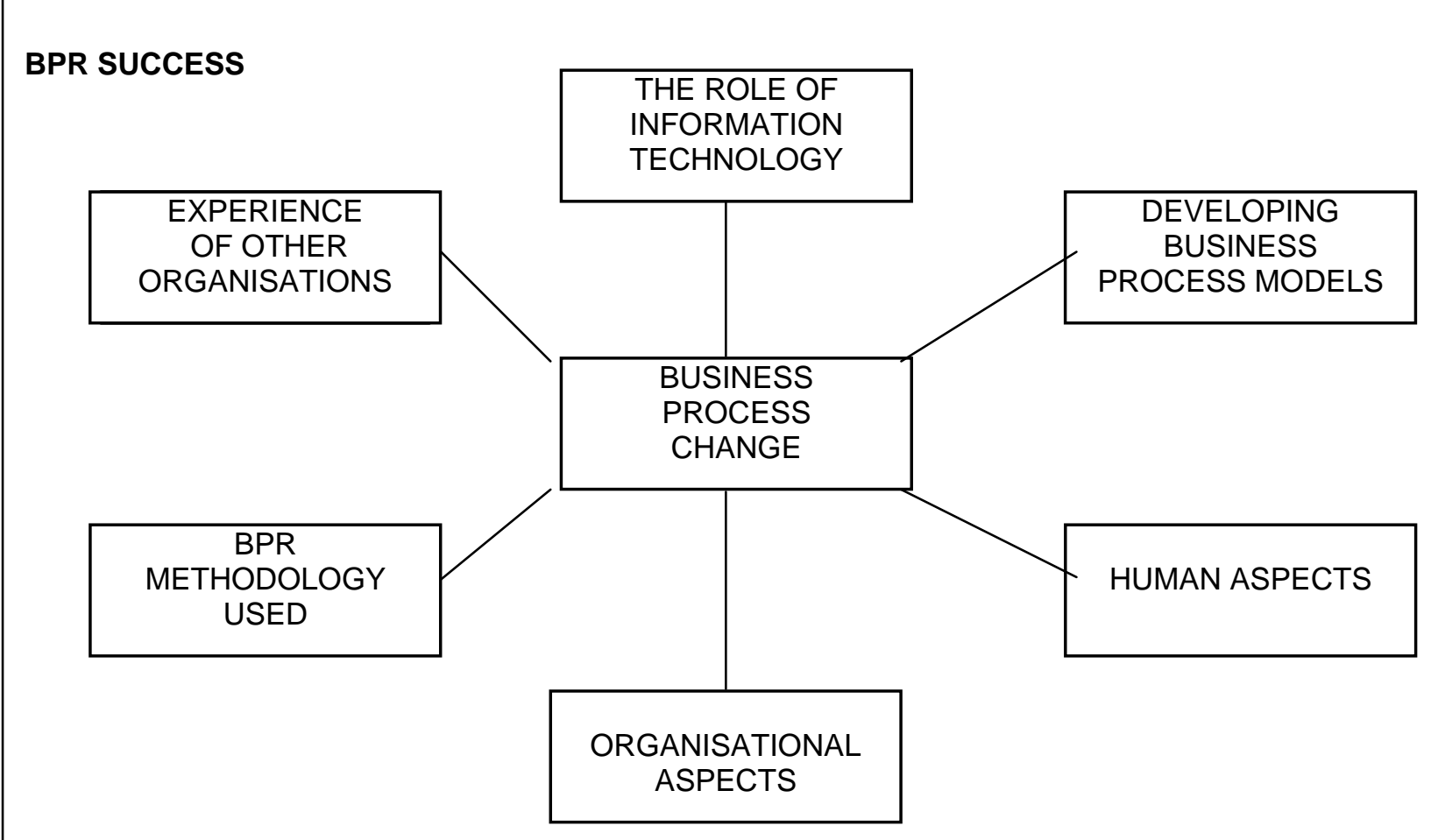


Figure 1 : REBUS Objectives

Figure 1 illustrates the REBUS approach to the success of BPR projects, emphasising factors that have to be considered in order to achieve successful BPR projects. For instance, appropriate BPR methodologies have to be applied. A methodology that includes a structured approach to BPR and emphasises a need to develop a model of business processes to be changed (so that the impact of changes and associated risks can be evaluated using this model) can improve the success of BPR projects. Experiences from other similar organisations that undertook BPR, investigating cases of BPR success and failure and learning lessons from other BPR projects represent another important area to be considered.

Additional factors that are vitally important to BPR projects are the human aspects. These factors could determine how the resistance to change could be reduced and how teams involved in BPR projects could be better motivated which eventually leads to better performance within the team and better results for the BPR project. Within the organisational aspects, a corporate climate, removals of hierarchical structures and different management styles have been foreseen as important factors crucial to the success of BPR projects. The REBUS approach to BPR success is distinctive in comparison to other approaches as it provides a systematic and interdisciplinary view of factors important for the success of BPR projects. A majority of other approaches focus on specific aspects of BPR such as organisational issues, the role of Information Technology or BPR methodology. 
Human factors in Business Process Re-engineering

The aim here is to investigate how human factors and the appropriate change management strategies can contribute to the success of BPR projects. Specific issues investigated include the motivation, commitment and training of human resources.

Business process simulation

The Centre investigates the suitability of simulation for modelling business processes in order to reduce the potential risks associated with BPR. Evaluating alternative processes using computer models before implementing the change in a real system does this.

Cases of BPR success and failure

This is related to investigating cases of BPR success and failure in order to identify common contributory factors to different project outcomes. Results from this research should assist in providing appropriate methods for undertaking BPR projects that will improve the chances for success.

The role of Information Technology in BPR

In order to improve work efficiency, the capabilities of Information Technology (IT) have to be applied to redesign business processes. It is apparent that there has been little uncovered about the relationship between IT and BPR, and so the Centre has targeted its efforts in this area.

Knowledge Management and BPR

Effective management of knowledge within organisations (including knowledge generation, codification and transfer) can have significant impact on business processes. Some projects related to this area have been carried out by the members of this Centre. 
Table 1: The main areas of research in REBUS

Table 1 illustrates the main areas of research within REBUS and the focus is on the particular factors that need to be considered for the successful implementation of any BPR project. For example, research into the human factors is important to determine how the resistance to change can be reduced and how teams involved in BPR can be better motivated. Organisational aspects would determine a corporate climate, hierarchical structure and management style, which are all important for BPR success. Business Process modelling is investigated with the aim of reducing risks associated with BPR projects. Cases of BPR success and failure are investigated in order to determine common problems that contribute to project failure. The role of Information Technology as a BPR enabler or implementor is another important area that is being investigated by the members of REBUS. It has been perceived that knowledge management is becoming increasingly important for organisations. Capturing tacit knowledge and disseminating it amongst employees has significant impact on business processes, organisational culture and general performance of a company. This is one of new areas being researched within the Centre,

Other research areas being investigated include: the study of interorganisational system stakeholders and the role of stakeholders in business process re-engineering (Pouloudi and Whitley, 1997), business process modelling, IS-enabled change management (Giaglis et al., 1999a) the success and failure of business reengineering in developing and industrial countries, the coherence of business process changes and Information Technology (Choudrie et al., 1998) and management innovation and change panaceas (Currie, ????). 
The following two sections provide examples of projects carried out by REBUS members. The first project relates to human and organisational aspects of BPR, where, in particular, the motivation of teams undertaking BPR is investigated. The second example relates to the business process-modelling project, where models of business processes were developed prior to change in order to reduce risks associated with change.

\subsection{Researching the human and organisational factors of BPR}

Analysis of the literature showed that the engineering aspects of BPR are well researched, but the human side of BPR has been virtually ignored (Oram and Wellins, 1996), a view supported by Corrigan (1996), who emphasises the importance of the role that people play in ensuring the success of BPR projects. Human factors such as empowerment, communication, and the selection criteria employed when forming the team can be seen as essential for the successful adoption and implementation of BPR initiatives. When an organisation undertakes BPR, radical changes in work areas, job preparation, peoples' roles, values and work units are apparent. As well as individuals, teams of people are viewed as important in the development and implementation of BPR. Without the reengineering teams, organisations would not be aware of the views and opinions held by members of the organisation regarding change, and would not know how to bring about the process change (Zuboff, 1998). Additionally, since teams rely on interdependence and synergy in order to function, increased productivity, contribution, learning and growth are all facilitated (Covey, 1997). The teams are also considered beneficial as they bring various stakeholders within an organisation together 
(Pouloudi and Whitley, 1997). Stakeholders have their own interests, and by committing themselves to the team, these can be reconciled. For instance, a case team could consist of technicians, managers, or consultants, each having experience and knowledge of different areas of expertise. Different areas can thus be reconciled by bringing them together as a team, and in doing so, enabling them to work towards a common goal.

There are many challenges involved in forming teams. One such challenge is that of team composition. Carr et al. (1996) suggest that a reengineering team must consist of the best people in the organisation. This is not altogether unsurprising, given that when putting together any team, whether for the next football match, or a project, a manager would be keen to choose the best people for the task. However, one of the difficulties in doing in such an instance is the selection of candidates when composing the team. There are instruments such as psychometric tests that have been developed or the traditional knowledge skills and attributes methods that could be utilised. Psychometric tests have been subjects of research for some time but the knowledge, skills and attributes area is still new (Hammer and Stanton, 1996), therefore, until then a large gap remains unfilled and the selection of teams remains an area that still requires the utmost attention. Theorists in the reengineering area, Hammer and Stanton (1996) suggest that a team member could be one who is dedicated to reengineering the process, to the needs of the customer, and to the team itself. However, the question that remains to be determined then is how would the dedication of the individuals be measured. If this is established, then the needs of the customer and team have to be recognised and according to them the selection process is undertaken. The biggest disadvantage in this case is whether the team member is suitable according to the selection methods utilised. Ultimately, the 
selected person has to function within a team and without a selection process, this weakness may go undeterred and ultimately, the required task may be unfinished and could lead to a failure within the BPR project.

Organisations also face resistance when forming teams. For example, Hammer and Stanton (1996), and Bennis and Mische (1995) found that employees are often resistant to change, and may act as a barrier to it. Interestingly enough, some individuals perceive a team environment as a demotion in job title (Kennedy, 1994). It was found that managers have conflicts with those at lower levels. With the hierarchical structure firmly embedded in their thinking, the managers were not willing to compromise their positions and work collaboratively and collectively as a team and thus conflicts arose. Conflicts in turn have a negative impact upon the motivation of the various individuals and this could affect the performance of the team and eventually the task at hand.

Another challenge is that of motivation. Some members of a team could be so dedicated to the team that their own daily tasks could be neglected (Hammer and Champy, 1995) and this might be detrimental to the success of the organisation. Since teams are composed of different individuals, varying motivating factors exist. For instance, a manager could be motivated by the possibility of promotion, or recognition amongst his/her peers, which may follow as a result of the success of a BPR project. On the other hand, a technician brought in to assist with the analysis of the process may not be rewarded as much as the manager, resulting in a loss of dedication to the project. In this case, the issue of motivation then depends upon the dedication of the individuals. However, even though the right incentives may exist, some teams still do not function effectively in the work environment. In such situations, organisations may form skunk 
workgroups (Harris, 1997) to spend time in external environments. These environments are meant to bring collectivism, concentration and commitment to the project within an isolated environment.

Another issue when forming teams is that of the type and amount of information available to team members (Oram and Wellins, 1996). Team members are receptive to change if information about the strategy or change is available. If organisations venture into the application of a strategy without full knowledge of the concept, this may cause problems, as further explanations to team members and management during progress meetings will not be provided and could demotivate team members.

By providing this normative information about the research area, it has been demonstrated that several factors including the selection process utilised in forming the teams, the type of information provided to the team and motivation are crucial for the success of a BPR project. Within a climate of high failure rates, it is important to glean insights into this vital area. However, the main question to be answered is what specific human factors will lead to the success of BPR projects, and this underpins much of the work done by the Centre.

\subsection{An example of the business process modelling project}

The objective of this project was to identify and re-engineer the processes that exist within the Telephony System of a large multi-national company. This was achieved by conducting surveys and interviews with key people within the system. The data gathered during this procedure was integrated in the form of Data Flow Diagrams that 
detailed the processes within the system and the information flowing between each. A number of processes along with the problems associated with each were identified.

These include Service Request/Enquiry (processes related to requests for a new services from employees), New Starter (processes ensuring that all required services are in place when the new employee joins the company), Paying for services (payment to providers of telephony services), Cost Centre (all employees belong to a Cost Centre according to the location they work in) and Write-off cars (processes in place when a car containing a car-phone has been written off). Analysis of these processes revealed that various problem areas existed. For example, it was found that Service Request/Enquiry process had too many groups involved - it was possible for three different groups to be involved when a request or enquiry was made, which was potentially confusing for the users unsure about whom to contact. Another problem was the lack of a standard order form, as the Information Systems Department (ISD) used a different order form to the Helpdesk when a service was requested. The result of this was the need for the Helpdesk to transfer relevant information from the ISD form to their form.

An additional problem that was identified was the deficiency of a clear understanding of the ownership of any process. Many groups were involved and it was unclear who owned and was responsible for the process. The process was also too complicated and lengthy. Once a process was selected for re-engineering, in this case, Service Request/Enquiry, a model was developed using Process Charter (Scitor Corporation, 1995) a business process modelling package. Figure 2 shows the resulting model. It is outside the scope of this paper to provide a detailed explanation of models developed, but is rather intended to illustrate the concept of business process modelling investigated by members of REBUS. Once the model was complete it was essential to 
ensure that it was a fair representation of the real process. This was accomplished by comparing the values obtained during observation and participation in the process and the values obtained from the model. The model was set-up to complete a cycle for one working day and executed five times with a different set of random numbers each time. In both cases, the model and real process, the average time per call was calculated and it was apparent that results from the model were relatively close to the real process. However, it was deemed necessary to evaluate the standard deviation which indicates just how close, to the average figure, each of the values actually were. Using these values it was possible to calculate a percentage which represents how close the calls from the model were to the calls from the real process. The results demonstrated that for each type of call the percentage ranges from $89 \%$ to $97 \%$.

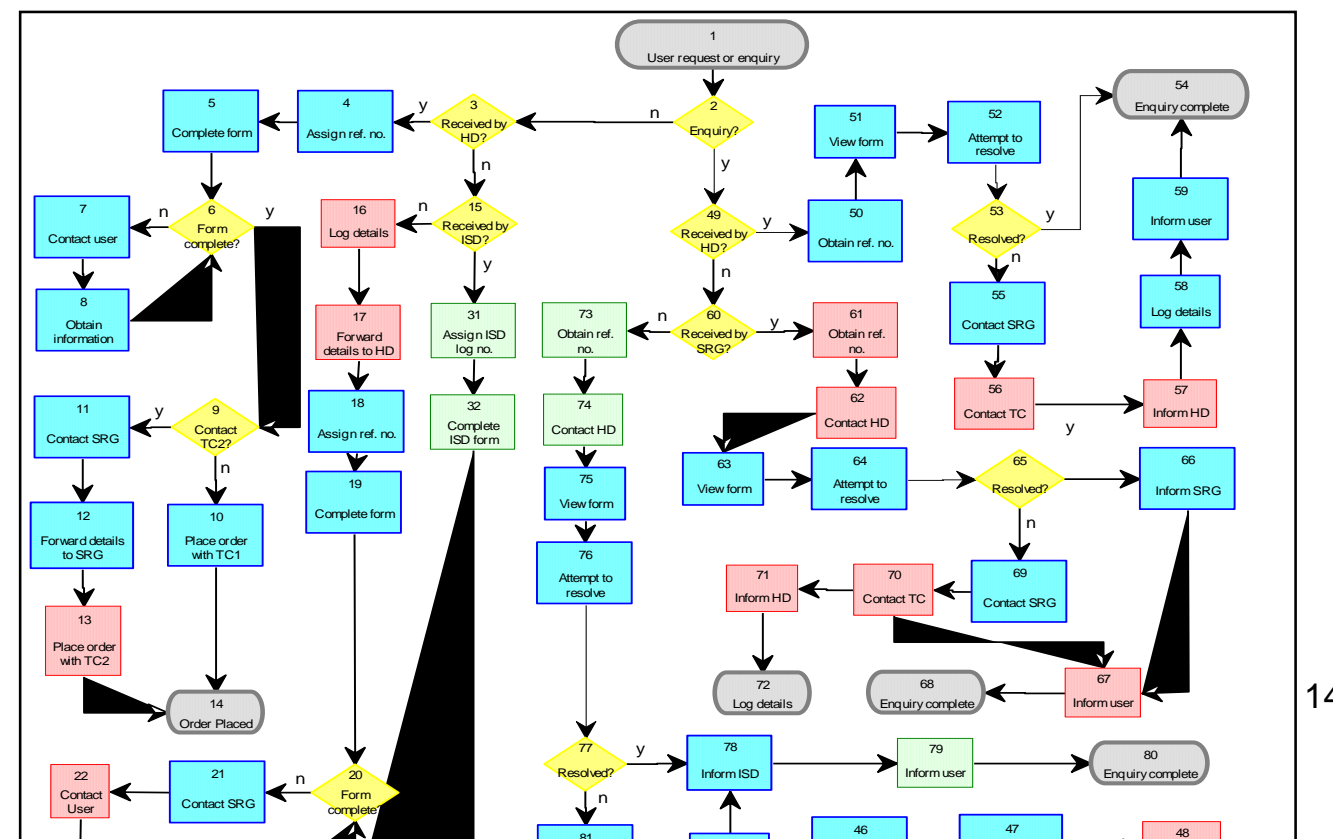


Figure 2 : Original model of the telephony system

After further analysis of the model and the results produced it was noticed that particular areas of the process were causing huge delays. For instance, contacting the user for further information could take any length of time, from 10 minutes up to 2 days, stalling the completion of the service request. Various experiments were conducted using this model in order to find out how the process could be made simpler and more efficient. Three alternative processes were proposed:

1. A Service Request/Enquiry was handled by the Helpdesk only, that is, no other groups were involved

2. A Service Request was handled by the Helpdesk and the Service Representatives Group was responsible for Service Enquiries 
3. The final alternative process ensured that when an order form entered the system, it had to be complete and therefore no contact with users was required - the Helpdesk controlled both Service Requests and Enquiries.

Models representing each alternative were produced and tested using the same test data used for the original model. Figure 3 shows models of the recommended alternative processes. The results from each model representing the alternative process along with the results from the model representing the original process were compared.

Results of the alternative process 1 , where the Helpdesk was responsible for dealing with all calls entering the system, demonstrated that there were significant improvements compared to the results from the original model and to the results from the other suggested recommendations. The models developed provided a better understanding of the processes involved and consequences of changes that the BPR approach would initiate.

Figure 3: Alternative processes

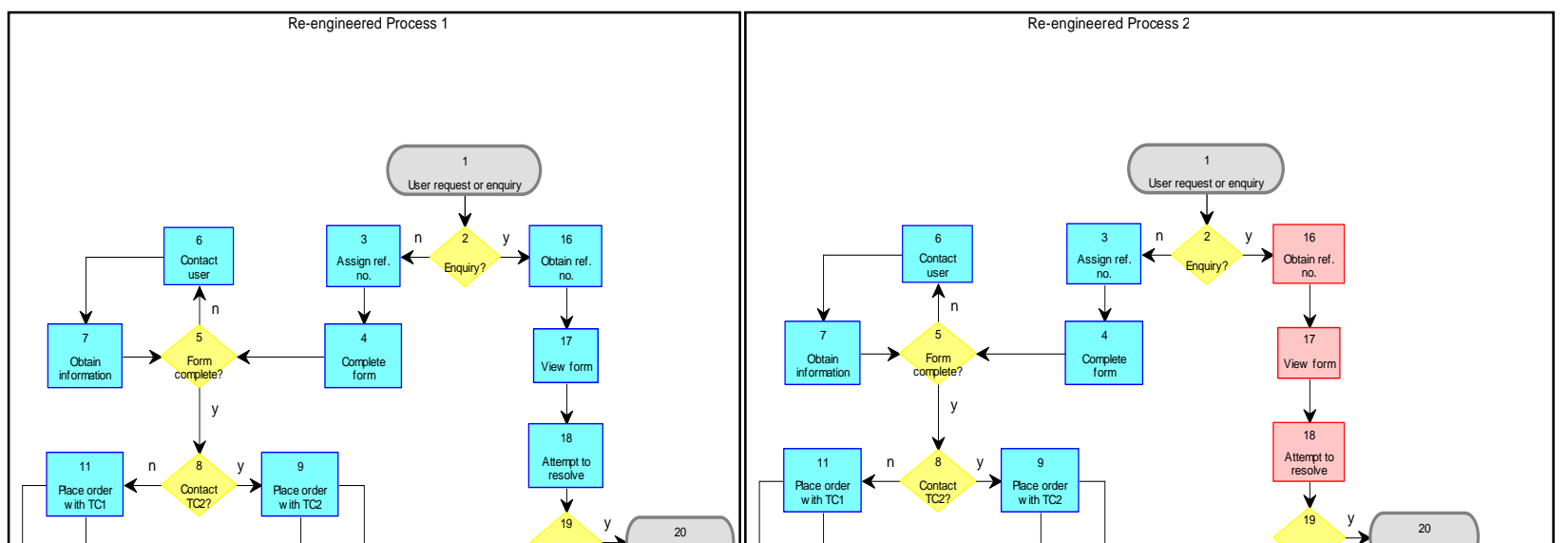




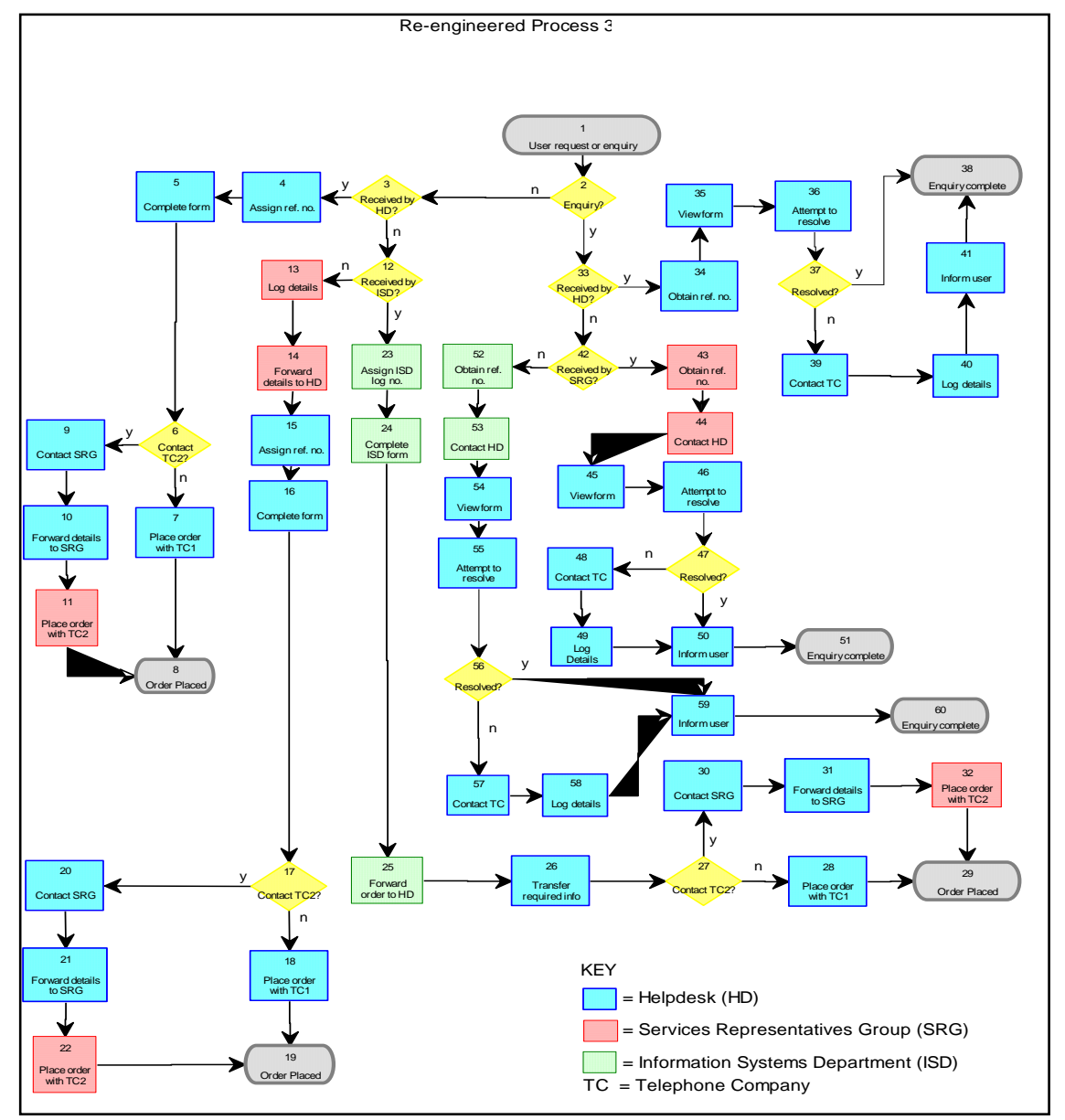

6.0 Experiences and conclusions 
The systematic and multidisciplinary approach to research in business process reengineering appears to be beneficial in improving the rate of success of BPR projects. When BPR projects are undertaken, various factors have to be considered such as the role of Information Technology in business process change, as well as human and organisational factors. The latter relate to, for example, resistance to change or motivation, and to company culture and management styles. Other important issues identified and requiring consideration is the utilisation of dynamic modelling techniques to develop models of processes prior to their change in order to evaluate risks and consequences of changes.

One of the future areas of REBUS research is likely to be in the area of integrating project management issues with BPR. In a report by ProSci (1997), investigators felt that education about project planning should be provided, such that those involved in the project would have something to look forward to rather than it being seen as a daily 'drudge'. It was also felt that projects would not have been abandoned or been condemned as 'failures' in such instances. If further investigation into integrating project management in BPR is carried out, such consequences might be avoided, and fewer costs forborne by organisations.

\section{References}

Bennis W. and M. Mische (1995). The 21st Century Organisation: Reinventing through Reengineering.. Pfeiffer \& Company. San Diego. 
Carr D., K. Hard, and W. Trahant (1996). Managing the Change Process. McGraw Hill. USA.

Choudrie, J., V. Hlupic, and Z. Irani (1999). Teams and their Motivation for BPR. Forthcoming in International Journal of Flexible Manufacturing Systems.

Choudrie J., V. Hlupic, and R. O'Keefe (1998). A Framework for Applying IT within the Grocery Retail Sector. In the Proceedings of the 11th International Bled Electronic Commerce Conference, "Electronic Commerce in the Information Society", Bled, Slovenia, June (1998), (Ed. by Doukidis G.J., Gricar J. and Novak J.), pp.372-386, Moderna Organizacija, Kranj.

Corrigan, S. (1996). Human and Organisational Aspects of Business Process Reengineering. Institute of Work Psychology, University of Sheffield.

Covey, S.R. (1997). The Seven Habits of Highly Successful People. Simon \& Schuster. London.

Currie W. Management Innovation and Change Panaceas: Strategic Vision or Tunnel Vision. Forthcoming in European Conference of Information Systems, Helsinki, Finland.

Giaglis, G.M. and G.I. Doukidis (1998). BPR in Support of Electronic Commerce: A Case Study. In Wanger, R.R. (Ed.), Proceedings of the 9th International Conference on Database and Expert Systems Applications, Vienna, August, IEEE Computer Society Press, pp. 655-660. 
Giaglis, G.M., N.A. Mylonopoulos, and G.I. Doukidis (1999a). Simulation Modelling for Information Systems Impact Measurement. Journal of Logistics and Information Management, 12, 1. Forthcoming.

Giaglis, G.M., R.J. Paul, and G.I. Doukidis (1999b). Dynamic Modelling to Assess the Business Value of Electronic Commerce, International Journal of Electronic Commerce. Forthcoming (January issue).

Giaglis, G.M., R.J. Paul, and V. Hlupic (1999c). Integrating Simulation in Organisational Design Studies. International Journal of Information Management. Forthcoming (April issue).

Giaglis, G.M., R.J. Paul, and R.M. O’Keefe (1999d). Integrating Business and Network Simulation Models for IT Investment Evaluation: A Research Note. Journal of Logistics and Information Management, 12, 1. Forthcoming.

Hammer, J. and J. Champy (1995). Reengineering the Corporation. HarperCollins Publishers. USA.

Hammer, M. and S. Stanton (1996). The Reengineering Revolution Handbook. HarperCollins Publishers. USA. 
Harris L. (1997). How You Can Develop a Skunk Works for Your Organisation. Proceedings of the Portland International Conference on Management of Engineering and Technology, Portland, Oregon, USA. July 27-31, 397.

Hlupic V. (1998). Business Process Re-engineering and Simulation: Bridging the Gap. In the Proceedings of the European Simulation Multiconference ESM'98, Manchester, United Kingdom, June, (Ed.by Zobel R. and Moeller D.), pp. 641-645, SCS.

Irani Z and J.M. Sharp (1997). Integrating Continuous Improvement and Innovation into a Corporate Culture: A Case Study. The International Journal of Technological Innovation and Entepreneurship (Technovation), 17(4): 199-206.

Irani Z, J.M. Sharp and M. Kagioglou (1997a). Communicating through self-directed work teams in a manufacturing environment. The Journal of Workplace Learning, 9(6): 199-205.

Irani Z., J.M. Sharp and M. Kagioglou (1997b). Human Factors in Manufacturing: Communicating Through Empowered Teams. In Proceedings of the 13th National Conference on Manufacturing Research, 9-11th September, University of Glasgow, Scotland, pp. 211-215.

Jackson B. (1996). Reengineering the sense of self: the manager and the management Guru. Journal of Management Studies. 33: 571-590. 
Kennedy, C. (1994). Re-engineering: The Human Costs and Benefits. Long Range Planning, 27: 64-72.

Oram. M. and R. Wellins (1996). Re-engineering's Missing Ingredient. Institute of Personnel and Development, London.

Paolucci E., F. Bonci and V. Russi (1997). Redesigning organisations through business process re-engineering and object-orientation. Proceedings of the European Conference on Information Systems, Cork, Ireland, 587-601.

Pouloudi A., and E.A. Whitley (1997) Stakeholder identification in inter-organisational systems: gaining insights for drug use management systems. European Journal of Information systems. 1-14.

ProSci. (1997). Best Practices in Business Process Reengineering and Process Design. ProSci.

Paul R.J., G.M. Giaglis and V. Hlupic. Simulation of Business Processes: A Review. Forthcoming in American Behavioural Scientist.

Scitor Corporation. (1995). User's Guide - Process charter for Windows.

Stevens, M.J. and M.A. Campion (1995). Staffing Work Teams: Development and Validation of a Selection Test for Teamwork Settings, Journal of Management, 1999, 207. 
Tumay K, Business Process simulation. Proceedings of the WSC'95 - Winter Simulation Conference, Washington DC, USA. 1995. Ed.by Alexopoulos A., Kang K., Lilegdon W.R. and Goldsman D. SCS. 55-60.

Zuboff, S. (1998). In the Age of the Smart Machine: The Future of Work and Power. Basic Books Inc. 